\title{
A REVIEW ON ANALYTICAL CHALLENGES IN MONITORING AND CONTROLLING GENOTOXIC IMPURITIES
}

\author{
VANDAMME S SUTNGA*, SELVAKUMAR K, RAJESH R
}

Department of Pharmaceutical Analysis, Acharya and BM Reddy College of Pharmacy, Bengaluru, Karnataka, India. Email: vandamesutnga@gmail.com

Received: 23 June 2020, Revised and Accepted: 03 August 2020

\begin{abstract}
Genotoxic impurities (GIs) are chemical agents that have a DNA-interaction characteristic which can ultimately lead to cancer. Their presence in various drug substances had driven various regulatory authorities to guide monitor, control, and to limit their level in various drug products. The objective of this article is to review the analytical approaches and challenges faced while accessing, monitoring, and controlling GIs in pharmaceuticals and also a brief explanation such as low limits of GIs, matrix interference, non-volatility, and environmental conditions encountered during the analysis of GIs are also discussed in this paper. At present, several modern analytical techniques are being used for the analysis of GIs such as high-performance liquid chromatography, liquid chromatography-mass spectrometry, and gas chromatography-mass spectroscopy that have high selectivity and sensitivity, but at the same time, many researchers have reported several challenges while using these techniques. Impacts of GIs are very important and various international organizations such as the World Health Organization have set out rules for regulating these chemicals. Hence, we can conclude that analytical approaches and their challenges are essential to understand because they play a key role to develop robust analytical methods.
\end{abstract}

Keywords: Column liquid chromatography, Genotoxic impurities, Analytical methods and challenges, Nitrosamines, World health organization.

(C) 2020 The Authors. Published by Innovare Academic Sciences Pvt Ltd. This is an open access article under the CC BY license (http://creativecommons. org/licenses/by/4. 0/) DOI: http://dx.doi.org/10.22159/ajpcr.2020.v13i10.38847

\section{INTRODUCTION}

\section{Genotoxic impurities (GIs)}

GIs are chemicals that have carcinogenicities due to their DNA-interaction characteristics, and no safe exposure limit or dose is believed to exist [1]. The major source of GIs is the starting material used during the synthesis of the drug products. Similarly, GIs may arise as intermediate, by-products excipients, and their contaminants. Furthermore, catalysts, solvents, and reagents during the synthesis process, may become a potentially genotoxic origin. The leachable or deteriorating products can also lead to impurities of the drug product during storage or transportation during exposure to light, air, or hydrolysis [2].

The impacts of chemical carcinogens in many nations are very important and international organizations such as the World Health Organization (WHO) have set out rules for regulating these chemicals. According to the international conference of harmonization, assessment of GIs in pharmaceuticals M7 guidelines, they are categorized into five classes [3].

- Class 1 - These impurities are recognized to be genotoxic, carcinogenic, and poses a serious threat or risk

- Class 2 - These impurities are known to be genotoxic but they do not have their carcinogenic potential. These impurities must, therefore, be controlled to some extent with the "Toxicological Threshold approaches (TTC)"

- Class 3 - These impurities have an alerting structure that is not related to the structure of the drug substances and the genotoxic potential is unknown. In these groups, the impurities are identified for the structure-activity relation

- Class 4 - These impurities shared a common parent's structure with that of the drug substances and have an alert functionality and are considered to be non-genotoxic

- Class 5 - There are no structural alerts to these impurities. These are regarded as non-mutagenic impurities.

GIs are controlled because they pose a cancer risk for human beings, and even low levels of such impurities can be of major toxicological concern in the final active pharmaceutical ingredient (API). It is, therefore, very important that GIs are identified in drugs and monitored at very low levels, to guarantee the safety of the community. The objective of this article is to review the analytical approaches and challenges to access, monitor, and control GIs in pharmaceuticals [4-6].

Several challenges have been encountered during the process of assessment of GIs. That includes detection, identification, quantification, and characterization of those GIs. There other factors such as various structural types of impurities, GIs may be unstable or chemically reactive. Hence, we have to adopt an approach or methodology or tool to identify, monitor, and control of GIs. Advanced instruments can help to reduce issues during detecting and can identify and measure a broader range of compounds and thus help to enhance data quality. Low levels of detection are also possible below the existing analytical check limit $[7,8]$.

\section{TTC APPROACH}

The TTC concept was used to determine GIs in drug formulation by a European Medicine Evaluation Agency (EMEA) board. A level of $1.5 \mu \mathrm{g} /$ day for GIs was proposed as acceptable limits. This strategy would be implemented in cases where there is no carcinogenicity information accessible in pharmaceutical products (e.g., Classes 2 and 3). A formula can calculate the limits of individual GIs $[9,10]$.

$$
\text { Limit }=1.5(\mu \mathrm{g} / \text { day }) \text { maximum daily dosage }
$$

In the EMEA guidance, the concept of staged TTC was outlined. The recommendations suggest that the main factor in cancer response is the duration of exposure. Table 1 suggests normal intakes of GIs.

\section{ANALYTICAL APPROACHES}

There are various analytical methods to measure trace levels of GIs for example high-performance liquid chromatography (HPLC), ultraperformance liquid chromatography, high-performance thin-layer chromatography, liquid chromatography-mass spectrometry (LC-MS), 
Table 1: Normal intakes of GIs [11]

\begin{tabular}{|c|c|c|c|c|c|c|}
\hline \multirow[t]{2}{*}{ Dose } & \multicolumn{6}{|c|}{ Duration of clinical exposure } \\
\hline & Single dose & $\begin{array}{l}>\text { Single dose } \\
\text { to } \leq 1 \text { month }\end{array}$ & $\begin{array}{l}>1 \text { month } \\
-\leq 3 \text { months }\end{array}$ & $\begin{array}{l}>3 \text { months } \\
-\leq 6 \text { months }\end{array}$ & $\begin{array}{l}>6 \text { months } \\
-\leq 12 \text { months }\end{array}$ & $\begin{array}{l}>12 \text { months or at } \\
\text { marketing }\end{array}$ \\
\hline Staged TTC $(\mu \mathrm{g} /$ day $)$ & 120 & 160 & 20 & 10 & 5 & 1.5 \\
\hline
\end{tabular}

TTC: Toxicological threshold approaches, GIs: Genotoxic impurities

gas chromatography-mass spectroscopy (GC-MS), and inductively coupled plasma mass spectrometry. These methods are recommended by the regulatory authorities to monitor the outcome of GIs [12]

\section{Advantages of analytical approaches}

1. Improving the process of synthesis and purification to eliminate the formation of impurity

2. Allowing a total daily exposure goal of a maximum of $1.5 \mu \mathrm{g} / \mathrm{day}$ of impurity

3. Assist genotoxic and carcinogenic threat to be calculated more effectively and enhance the adequate identification of impurities to larger or smaller rates

There are numerous methods available but only some of the most commonly used methods will be discussed.

\section{HPLC}

HPLC is regularly used to detect impurities in both bulk drugs and finished drug products. HPLC can provide diagnostic data regarding the impurity structure. HPLC offers a broad range of distinctive column packaging materials, a broad variety of detection methods, and solvent choices to provide a broad selectivity for separation. At present, HPLC is integrated with several detectors such as ultraviolet (UV), fluorescence, infrared, refractive index, evaporative light scattering, and mass spectrometry for identifying and elucidating the structure of impurities. Because of its ease and accessibility, HPLC with UV detection should be regarded as the first option for GIs assessment [13].

In the literature, several HPLC methods have been testified for the analysis of GIs. For instance, Wang et al. (2016) reported a simple and sensitive reversed-phase (RP) liquid chromatography method to determine hydrazine trace quantity in pharmaceutical materials with UV detector [14]. Soni and Sanjay developed an RP-HPLC method for the estimation of meloxicam and paracetamol with its GIs in bulk and combined dosage form using photodiode-array detection detector [15]. Dousa et al. reported an RP-HPLC method for the analysis of GIs in the vortioxetine using a fluorescence detector [16].

With HPLC, the process can be optimized using different sorts of columns, length, diameter, and particle size to guarantee that impurities are detectable. Commercial software programs can also be used to help optimize chromatographic parameters to ensure the separation of impurities [17].

\section{CHALLENGES IN THE HPLC METHOD OF ANALYSIS}

The HPLC method for the study of GIs faces various challenges. Development of HPLC methods is a long and complex process, frequently involving combining screening of columns and eluents, mobile phase and temperature optimization, the use of stationary selectivity, and the analysis of many representatives and stressed samples. Other factors such as $\mathrm{pH}$, organic modifier, and ionic additives are often of similar significance during the progress of chromatographic methods, although the selection of a stationary phase is the key. These variables result in a huge amount of experimental circumstances making the strategy of the technique a challenge $[18,19]$. Another major challenge is with the detectors, for example, some impurities cannot be identified by the use of UV detectors due to the lack of UV absorbing chromophores. The impurities must be derivatized with a colored complex to solve this issue. In some cases, if the UV spectrum of an unknown impurity is comparable with the drug material, the impurity is likely similar to that of the drug substance for drug-related impurities and there is a probability that the impurity has the same chromophore as the drug substances [20]. Reddy et al. stated that trace level analysis, HPLC with UV detection may not give adequate sensitivity to certain GIs and when chromophores are lacking in GIs; the alternative option is evaporative light-scattering detection. The other alternative detectors used in HPLC are a refractive index detector and a fluorescence detector. In fluorescence detector compounds must contain innate or natural radiation for good detection or if there is no visible radiation, labeling with fluorescent tags will be required [21]. Al-Sabti et al., during their work on the analysis of $\mathrm{N}, \mathrm{N}$-dimethylaniline reported that this impurity is a small polar molecule; hence, it cannot retain in the stationary phase so to overcome this they have employed hydrophilic interaction liquid chromatography mode which shows good results both for separation and quantification of this impurities [22]. Anerao et al. (2020) reported solubility and matrix issues while analyzing 1-Naphthol and 1, 2-Dichlorobenzene which ultimately affect the specificity of the method [23]. Landge et al. reported HPLC analysis of structurally similar impurities in vortioxetine hydrobromide drug and they have stated that choice of column and $\mathrm{pH}$ modifier is important to prevent coelution of peaks and enhanced the resolution of the method [24]. Senthil et al. reported an analysis of 4, 4- BIS (Bromomethyl) Biphenyl GIs by HPLC, in their findings they have stated that column types, choice of solvents and temperature is a challenge to achieve a reproducible result [25]. The analytical challenge in HPLC method is given in Table 2.

\section{LC-MS}

LC-MS is a blend of two selective techniques that make it possible to isolate and measure analytes of interest in highly complex mixtures. LCMS differentiate compounds by their mass to charge ratio ( $\mathrm{m} / \mathrm{z}$ ratio). At present, LC-MS has been widely used for GIs analysis because of its high selectivity and sensitivity. LC-MS based methods provide additional robustness and ruggedness due to their high specificity and sensitivity [26].

Compounds that lack the chromophoric group can also be analyzed using LC-MS. It can, therefore, regard as the universal detector for pharmaceutical sample analysis. LC-MS provides spectral data that generate useful information on molecular weight, identity, purity, quality, sample composition, and structure [27].

Volatile buffers should be used for an effective LC-MS solution. Volatile ammonium and ammonium acetate are the most appropriate buffers for LC-MS analysis. The first step in mass is that the sample needs to be ionized. The analytes are transformed into fragmenting ions in the ionization process. The selection of the ionization techniques depends on the state and nature of the sample. Some of the commonly used ionization techniques include electron-impact (EI), atmospheric chemical ionization (APCI), electron spray ionization (ESI), matrixassisted laser desorption ionization, fast atom bombardment, and $\mathrm{CI}$, respectively. According to the research techniques such as ESI and APCI are mostly used because of less fragmentation of parent, ions compare to other techniques.

ESI technique is a soft ionization technique; the method generates ions from a solution of a sample by creating a fine spray of charged droplets. The sample solution is sprayed across a potential difference 
from a needle into an orifice in the interface. The charged droplets are then passed to a desolvating tube and the solvents get evaporated with the help of a vacuum. The ions then move toward the analyzer. The benefit of ESI is the retention in the gas phase of solution-phase data. However, the sample mass range contains little structural data. ESI can be coupled to tandem mass spectrometry (ESI MS/MS) to overcome this problem [28].

Similarly, APCI is a soft ionization process, which sprays the solution into the heater around to vaporize the sample molecule and the solvent. The solvent molecule is then ionized to produce stable reaction ions with a corona discharge. APCI is also well suited for small, slightly polar to non-polar molecules. This method can also be used by molecules that are not completely ionized by ESI. Once the samples have been ionized it is then passed through the mass analyzer where the sample separates them according to the mass to charge ratios. Some example of mass analyzers includes quadrupole, time of flight (TOF), quadrupole-TOF (Q-TOF), and ion-trap mass analyzer [29].

Quadrupole mass analyzer consists of four parallel rods placed opposite to each other. A pair of rods is fitted with an radio frequency (RF) voltage and a pair with a direct current (DC) voltage has been fitted. In any given $\mathrm{DC}$ and RF combination, only the ions of a specific massto-charge ( $\mathrm{m} / \mathrm{z}$ ratio) can pass through the analyzer, while the analyzer does not pass other ions with unstable trajectory, since their oscillating amplitude is infinite. Usually, ions with different $\mathrm{m} / \mathrm{z}$ values may be passed to the detector one by one by changing DC and RF in time at a fixed ratio. TOF analyzer measures the flight time that the ions with the specific mass to charge have to reach the detector placed at a distance, accelerated by potential voltages. It is based on kinetic energy and ion velocity. Even though all ions have the same kinetic energy, the distance they travel to the detector along the flight tube is proportional to the mass of the ions [30].

There are several methods reported which utilized different ionization techniques. For instance, Szekely et al. reported a method for the trace analysis of 4-dimethylamino pyridine by LC-MS using the ESI technique and a quadrupole analyzer [31]. Venugopal et al. reported an LC/MS method for the trace analysis of GIs (2 chloromethyl-3,4-dimethoxy pyridine hydrochloride) in pantoprazole sodium drug substances using ESI technique [32]. Chen et al. reported an LC-MS method for analyzing potential GIs in pantoprazole [33].

\section{CHALLENGES IN LC-MS ANALYSIS}

Both LC and MS can be hard to optimize. The ionization mechanism can be particularly complicated. During the ionization of compounds, several species are formed and multiple charging can occur. Conditions for optimal sensitivity and reproducibility must be selected with care. Besides ionization, several others such as matrix effect which impacts all other sample parts, except for the particular compound to be quantified on an analytical method but a greater incidence of matrix effects in methods for LC-MS has led in a better understanding of the factors that have contributed to these effects. Several approaches have been studied to increase the productivity and robustness of matrix-subject LC-MS techniques. Regular maintenance and additional costs are also needed in LC-MS to guarantee system stability; environmental circumstances in the laboratory must be well controlled. Another challenge encountered

\section{Table 2: Analytical challenges in HPLC method}

\begin{tabular}{ll}
\hline Problems & Sources \\
\hline Variable & Changes in mobile phase composition, trapped air \\
retention times & inside columns, overloading of column \\
Selectivity & Changes in the ionic strength or pH \\
UV detectors & Absence of absorbing chromophore \\
Fluorescence & Absence of fluorescence \\
detector & \\
\hline
\end{tabular}

HPLC: High-performance liquid chromatography, UV: Ultraviolet is retention time. LC retention time may differ, and some methods are required to characterize impurities online when there are no impurity standards available.

Many authors have expressed the challenges they come across while working with LC-MS, for instance, David et al. (2009) demonstrate a practical example for the analytical control of five GIs in the manufacturing process of pazopanib hydrochloride an anticancer drug by LC-MS method using ESI operated in positive ion mode. The author stated that these hyphenated MS-based trace analysis methods are nonroutine, costly, and difficult to implement in quality control laboratories in a manufacturing environment; a strategy to simplify the analytical testing is, therefore, imperative [34].

Although the regulatory agencies is concerned about the trace level of GIs in drug substances. However, specific guidance is currently lacking in how to cope with this challenge. If it is not tackled strategically, it may ultimately impede productivity and inflate costs by drawing extensive analytical resources into drug development and manufacturing. David et al. (2010) reported that LC-MS does not provide a solution due to low retention, ionization suppression, low response, possible GIs, and/ or API hydrolysis in aqueous or mobile solutions, etc. [35]. Van et al. (2011) stated that the implementation of LC-MS may be constrained by the characteristics of the compound and matrix. While derivatization in the pharmaceutical analysis is ideally avoided, it can be used to circumvent specific limitations, such as poor sensitivity in LC-MS analysis [36]. Suryakala et al. reported that the stability and sensitivity of the method are affected by sample preparation during the analysis of GIs, the author's claims that using a technique called selective ion monitoring (SIM) mode, we can eliminate matrix interference which drastically enhances the accuracy, quantification, and detection limits [37]. The problems with LC-MS is given in Table 3.

\section{GC-MS}

GC-MS is used most frequently to identify unstable, semi-volatile, and thermally stable compounds, residues, and solvents. In GC, the separation method is focused on conditions such as the size of the column, the temperature of the column, the type of carrier gas used, and the analyte characteristics such as vapor stress and polarity. For better separation, the analyte must have significant vapor pressure between $30^{\circ} \mathrm{C}$ and $300^{\circ} \mathrm{C}[38]$.

The direct injection technique is the most frequently applied in GCMS this is achieved by combining split and splitless injectors. Through splitting mode, a part of the sample is passed into the column and the remainder is directed to waste. In splitless injection, the split vent is shut for a normal duration of 0.5-1 min and the column oven temperature is set at least $10^{\circ} \mathrm{C}$ less than the injection solvent boiling point. It allows the analytes to condense into a tight band on the head of the column. The split valve will be opened after the split time to flush the injection of any residual specimen [39]. Another injection method that is beneficial to eliminate injector or column contamination is used this is called headspace injection. In this case, the sample is dissolved in dimethyl sulfoxide (DMSO) and the sample solution is enclosed in

\section{Table 3: Problems in LC-MS}

\begin{tabular}{|c|c|}
\hline Problem & Sources \\
\hline Excessive & Apart from the specific analyte, there can be \\
\hline Selectivity & $\begin{array}{l}\text { many other components present which can create } \\
\text { problems with quantitation }\end{array}$ \\
\hline $\begin{array}{l}\text { Limited } \\
\text { dynamic range }\end{array}$ & $\begin{array}{l}\text { The range should not exceed a } 500 \text {-fold } \\
\text { concentration }\end{array}$ \\
\hline Matrix effect & $\begin{array}{l}\text { Disturbance arising from other components of the } \\
\text { sample }\end{array}$ \\
\hline Poor precision & $\begin{array}{l}\text { Unwanted components coelute with the analyte of } \\
\text { interest }\end{array}$ \\
\hline
\end{tabular}


a headspace vial [40]. The separated components are then passed to mass spectroscopy for detection. EI and CI are commonly employed in ionization modes in GC [41].

In the literature, there are methods reported which use different injection techniques, for example, Wollein and Schramek reported a GC-MS method for the concurrent analysis of methyl mesylate, ethyl mesylate, isopropyl mesylate, methyl besylate, and ethyl besylate using direct injection techniques [42]. Ho et al. reported a method for the determination of alkyl/aryl halides and nitro-aromatics GIs, in molecular drugs by GC-MS [43]. Raghavender et al. (2018) reported a GC-MS method for the analysis of five GIs methyl bromide, ethyl bromide, isopropyl bromide, $n$-propyl bromide, and $n$-butyl bromide in Divalproex sodium drug substance [44].

\section{CHALLENGES IN GC-MS ANALYSIS}

The handling of samples is a difficult task in GC-MS. Before analysis, the sample requires volatilization, which may cause problems related to chemical degradation and new product formation under high heating conditions. On the other hand, the volume of samples, large quantity of a drug substance makes the evaluation of derivatives by direct injection GC impractical, as very often methods suffer from contamination-related problems of robustness. Safety is another significant concern, highly boiling organic diluents causing safety problems and also affecting the overall sensitivity of the method, for example, DMSO can significantly increase the internal pressure in reaching boiling points and break up the vial septa or rupture the vial itself. Some authors, for example, Chen et al. reported that diluent plays an important role during sample preparation in GC-MS so as proper peak shape and good recoveries can be achieved. For example, during the analysis of epoxide GIs, DMSO had the matrix effect of a tailing peak that could increase the response of the SIM signal. The possible explanation is that DMSO's boiling temperature is high and similar to that of the GIs, triggering the effect of the diluent matrix. Furthermore, the decomposition of the API is a serious concern since it induced matrix interference with the GIs signal. Setting the inlet temperature too high can lead to thermal decomposition of the analytes and matrix [45]. Ahirrao et al. reported a GC-MS method for the estimation of GIs in a new antibacterial agent using selective reaction monitoring (SRM) tool instead of SIM. Mass spectrometer scans all SRM simultaneously they have stated that simultaneous scanning of multiple transition cause lowering of sensitivity but using SRM mode made the method more specific and selective compared to SIM mode [46]. Anerao et al. reported that if a large volume of a test sample is introduced into the GC column it can get damage in the long run so to overcome this problem headspace injector is preferred. They also reported in their finding that the sensitivity of a flame ionization detector is not sufficient while analyzing impurities at a low concentration which creates a challenge when analyzing some impurities to overcome this problem pre-column derivatization technique is required before carrying out the analysis [47]. The problems in GC-MS analysis is given in Table 4 .

\section{OTHER CHALLENGES DURING THE ANALYSIS OF GIS}

Other difficulties in evaluating GIs include numerous structural forms of GIs that require different methods and techniques. Many GIs are non-volatile, highly reactive, acidic, and non-chromophoric causes a problem for analysis and identification in drugs. Changes in environmental conditions might affect the stability of GIs. When using MS for analyzing GIs, the analytical intermediates API act as a matrix that interferes and affects accurate measurements through ion suppression. Low limits conjointly create a challenge because GIs have low limit supported dose and exposure period. A pre-concentration phase of GIs was also required before chromatographic separation due to their low concentrations.

The sensitivity range required to analyze GIs is $\mathrm{ng} / \mathrm{ml}$ range for drug concentrations in several $\mathrm{mg} / \mathrm{l}$ range or $\mathrm{ppm}$. The authorities have set up limits to determine the allowable daily intake of GIs based on the TTC level or TTC of $1.5 \mu \mathrm{g} /$ day. Most of the analytical techniques can
Table 4: Problems in GC-MS

\begin{tabular}{ll}
\hline Problems & Sources \\
\hline Robustness issues & $\begin{array}{l}\text { Potential interferences resulting } \\
\text { from large quantities of drug } \\
\text { substances and their impurities } \\
\text { Because of high reactivity and low }\end{array}$ \\
$\begin{array}{l}\text { Poor recovery, improper peak } \\
\text { shape, and separation }\end{array}$ & $\begin{array}{l}\text { response by detectors } \\
\text { A large amount of non-volatile } \\
\text { analyte. }\end{array}$ \\
$\begin{array}{l}\text { Trace analysis of GIs using } \\
\text { flame ionization detectors } \\
\text { performance not satisfactory }\end{array}$ & $\begin{array}{l}\text { analyte } \\
\text { GC-MS: Gas chromatography-mass spectroscopy }\end{array}$
\end{tabular}

GC-MS: Gas chromatography-mass spectroscopy

provide this level of sensitivity but it is also necessary that the sensitive method also be highly selective. The selective and sensitive method must also be able to support the testing of products from the early development of the impurities to post-development. After the drug has been marketed, it is important to conduct this level analysis. This is a major challenge for the pharmaceutical industry because the method must also be robust to successfully execute marketed product testing sites around the world [21].

\section{ANALYTICAL CHALLENGES FOR NITROSAMINE IMPURITIES}

According to the WHO nitrosamines or more specifically N-nitrosamines are molecules that contain a nitro functional group. These molecules are carcinogenic to humans. Nitrosamines also increase cancer risk when people are exposed to them. The WHO stated that nitrosamines are found to be present in some food and drinking water but their presence in medicinal products is deemed unacceptable [48]. The recent findings of nitro dimethylamine pose a serious issue in the drug industry which made the regulatory bodies fully conscious about the existence of nitrosamine impurities in drug products. Recent druglike pioglitazone and ranitidine have been reported to contain these nitrosamine impurities. As stated by the (EMA) sartans that have a tetrazole ring contain nitrosamine impurities. In non-sartan drugs, the nitrosamines can form depending on the manufacturing of the drug products. Nitrosamines are reported to be found from certain solvents, recycled reagents, or raw materials; contaminated equipment used during the manufacturing process. Authorities have demanded that the level of these impurities should be controlled to certain acceptable limits $[49,50]$.

Some of the challenges researchers faced while analyzing nitrosamines impurities are low-level detection because of matrix interference, method development and standardization, extraction and derivatization of the compounds before analysis, $\mathrm{pH}$, sensitivity, and availability of instruments such as LC-MS. For instance, Jeffery et al. (2004) report that GC-MS with EI lacking selectivity and producing potentially non-distinctive patterns of fragmentation. High-resolution mass spectrometry can be used in low-resolution mass spectrometry to compensate for the lack of EI selectivity [51]. Hitoshi et al. (2018) reported HPLC separation with an inline photochemical reactor (PR) and subsequent luminol chemiluminescence detection posed a challenge for analyzing nitrosamines in water due to the high eluent $\mathrm{pH}(>10)$ needed for a photochemical reaction which limited the sensitivity and complicated the analysis. To challenge can be overcome by the addition of an anion exchange module to the HPLC system [52]. Wayne et al. (2002) reported the chemical diversity of the impurities results in very low retention and overall analysis time. The author recommended the addition of an ion-pairing agent to the mobile phase. For compensating this effect, and increasing the retention time of these compounds [53]. Yichao et al. (2014) stated that thermally unstable nitrosamines cannot be analyzed by GC-MS methods, for example, because of thermal decomposition at the injection port. Using HPLC-MS techniques can solve that problem [54]. Benigo et al. (2020) reported that due to the lower molecular weight of nitrosamines background 
ions can interfere with their determination and to overcome this problem application of high-resolution MS such as the incorporation of analyzer such as Q-TOF is recommended [55].

\section{CONCLUSION}

GIs are a significant factor in the development of both active pharmaceutical ingredients and pharmaceutical products. GIs are unpredictable, and poor sensitivity and low recovery can be a problem. However, some analytes lack structural features that correspond to commonly used detectors. Developing sensitive analytical methods to estimate GIs at very low levels is a major challenge due to this reason selective and responsive analytical methods are required to distinguish interference from APIs. Therefore, the molecular structure, properties of GIs, and challenges are essential to understand, because they play a key role to develop robust analysis methods.

\section{AUTHORS' CONTRIBUTIONS}

All authors conceived and collaborated. The author KS has drawn up the paper's first draft. Author RR handled the review of the literature. The author VSS has drawn up the paper's final draft. The final version of the paper was approved by all authors.

\section{CONFLICTS OF INTEREST}

All the authors declared that they have no conflicts of interest.

\section{AUTHORS' FUNDING}

Nil/Self.

\section{REFERENCES}

1. Liu DQ, Sun M, Kord AS. Recent advances in trace analysis of pharmaceutical genotoxic impurities. J Pharm Biomed Anal 2010;51:999-1014.

2. Qiu F, Norwood DL. Identification of pharmaceutical impurities. J Liq Chromatogr Relat Technol 2007;30:877-935.

3. Committee for Human Medicinal Products. ICH Guideline M7 (R1) on the Assessment and Control of DNA Reactive (Mutagenic) Impurities in Pharmaceuticals to Limit Potential Carcinogenic Risk. Geneva, Switzerland: International Conference on Harmonisation; 2015. p. 1-110.

4. Leistner A, Haerling S, Kreher JD, Becker I, Jung D, Holzgrabe U. Risk assessment reports of potential impurities in cetirizine dihydrochloride. J Pharm Biomed Anal 2020;189:1-13.

5. Holm R, Elder DP. Analytical advances in pharmaceutical impurity profiling. Eur J Pharm Sci 2016;87:118-35.

6. Pounikar AR, Umekar MJ, Gupta KR. Genotoxic impurities: An important regulatory aspect. Asian J Pharm Clin Res 2020;13:10-25.

7. Rahman N, Azmi SN, Wu HF. The importance of impurity analysis in pharmaceutical products: An integrated approach. Accredit Qual Assur 2006;11:69-74.

8. Kasper P, Muller L. Genotoxic impurities in pharmaceuticals. Genotoxicity Carcinog Test Pharm 2015;2015:55-74.

9. Munro IC, RenwickAG, Danielewska NB. The threshold of toxicological concern (TTC) in risk assessment. Toxicol Lett 2008;180:151-6.

10. European Medicines Agency. Guidelines on the Limit of Genotoxic Impurities, CPMP/SWP/5199/02, EMEA/CHMP/QWP/251344/2006. European Medicines Agency; 2006. Available from: https://www. ema.europa.eu/en/documents/scientific-guideline/guideline-limitsgenotoxic-impurities_en.pdf. [Last accessed on 2020 Jun 21].

11. Kroes R, Kleiner J, Renwick A. The threshold of toxicological concern concept in risk assessment. Toxicol Sci 2005;86:226-30.

12. Raman NV, Prasad AV, Reddy KR. Strategies for the identification, control, and determination of genotoxic impurities in drug substances. $\mathrm{J}$ Pharm Biomed Anal 2011;55:662-7.

13. Yuabova ZY, Holschlag DR, Rodriguez SA, Qin C, Papov VV, Qiu F, et al. Genotoxic impurities: A quantitative approach. J Liq Chromatogr Relat Technol 2008;31:2318-30.

14. Wang J, Yang S, Zhang K. A simple and sensitive method to analyze genotoxic impurity hydrazine in pharmaceutical materials. J Pharm Biomed Anal 2016;126:141-7.

15. Soni LK, Sanjay J. Optimization and validation of RP-HPLC method for the estimation of meloxicam and paracetamol with its genotoxic impurity ( $\mathrm{p}$-amino phenol) in bulk and pharmaceutical drug product using PDA detector. Asian J Biomed Pharm Sci 2016;6:21-6.

16. Dousa M, Doubsky J, Srbek J. Utilization of photochemically induced fluorescence detection for HPLC determination of genotoxic impurities in the vortioxetine manufacturing process. J Chromatogr Sci 2016;54:1625-30.

17. Olsen BA, Castle BC, Myers DP. Advances in HPLC technology for the determination of drug impurities. Trends Anal Chem 2006;25:796-805.

18. Teasdale A, Elder DP. Analytical control strategies for mutagenic impurities. Trends Anal Chem 2018;101:66-84.

19. Raman NV, Prasad AV, Reddy KR. Sensitive derivatization methods for the determination of genotoxic impurities in drug substances using hyphenated techniques. J Pharm Biomed Anal 2014;89:276-81.

20. Reddy AV, Jaafar J, Umar K, Majid ZA, Bin AA, Talib J, et al. Identification, control strategies, and analytical approaches for the determination of potential genotoxic impurities in pharmaceuticals. J Sep Sci 2015;38:764-79.

21. Mattrey FT, Makarov AA, Regalado EL, Bernardoni F, Figus M, Hicks MB, et al. Current challenges and future prospects in chromatographic method development for pharmaceutical research. Trends Anal Chem 2017;95:36-46.

22. Al-Sabti B, Harbali J. Trace analysis of potential genotoxic impurity N, $\mathrm{N}$-dimethylaniline in linagliptin active pharmaceutical ingredient using HPLC. Sep Sci Plus 2020;3:1-7.

23. Anerao A, Solase V, Gadhave T, More A, Pradhan N. Quantification of genotoxic impurities in key starting material of sertraline hydrochloride by simple and sensitive liquid chromatography technique. Curr Pharm Anal 2018;16:110-6.

24. Landge SB, Dahale SB, Devadhe SJ, Desmukh DG, Solanki PV, Jadhav SA, et al. Separation and quantification of structurally similar impurities by HPLC method of vortioxetine hydrobromide: An antidepressant drug. Eur Chem Bull 2020;9:114-8.

25. Senthil KS, Srivastava RK, Srinivas RV. Determination of 2-cyano-4'bromomethyl biphenyl genotoxic impurity in irbesartan drug substances using HPLC technique. Int J Pharm Pharm Sci 2016;8:225-30.

26. Kumar T, Ramya M, Srinivasan V, Xavier N. A simple and direct LC-MS method for determination of genotoxic impurity hydroxylamine in pharmaceutical compounds. J Chromatogr Sci 2017;55:683-9.

27. Singh S, Handa T, Narayanam M, Sahu A, Junwal M, Shah RP. A critical review on the use of modern sophisticated hyphenated tools in the characterization of impurities and degradation products. J Pharm Biomed Anal 2012;69:148-73

28. Lee H, Shen S, Grinberg N. Identification and control of impurities for drug substance development using LC-MS and GC-MS. J Liq Chromatogr Relat Technol 2008;31:2235-52.

29. Devanshu S, Rahul M, Annu G, Kishan S, Anroop N. Quantitative bioanalysis by LC-MS/MS. J Pharm Biomed Sci 2010;7:1-8.

30. Lee MS. LC-MS Applications in Drug Development. United States: Wiley, Interscience Series on Mass Spectrometry; 2002. p. 39-41.

31. Szekely G, Henriques B, Gil M, Ramos A, Alvarez C. Design of experiments as a tool for LC-MS/MS method development for the trace analysis of the potentially genotoxic 4-dimethylamino pyridine impurity in glucocorticoids. J Pharm Biomed Anal 2012;70:251-8.

32. Venugopal N, Vijaya BR, Gangadhar RK, Madhavi V, Madhavi G. Method development and validation study for the quantitative determination of 2-chloromethyl-3,4-dimethoxy pyridine hydrochloride a genotoxic impurity in pantoprazole active pharmaceutical ingredient by LC-MS/MS. J Pharm Biomed Anal 2012;70:592-7.

33. Chen Y, Wu S, Yang Q. Development and validation of LC-MS/MS for analyzing potential genotoxic impurities in pantoprazole starting materials. J Anal Methods Chem 2020;2020:1-8.

34. Liu DQ, Chen TK, McGuire MA, Kord AS. Analytical control of genotoxic impurities in the pazopanib hydrochloride manufacturing process. J Pharm Biomed Anal 2009;50:144-50.

35. David F, Jacq K, Sandra P, Baker A, Klee MS. Analysis of potential genotoxic impurities in pharmaceuticals by two-dimensional gas chromatography with deans switching and independent column temperature control using a low-thermal-mass oven module. Anal Bioanal Chem 2010;396:1291-300.

36. Van WA, Niederlander HA, Siebum AH, Vervaart MA, De JG. A new derivatization reagent for LC-MS/MS screening of potential genotoxic alkylation compounds. J Pharm Biomed Anal 2013;74:133-40.

37. Suryakala D, Susarla S, Rao BM. LC-MS method development for the quantitation of potential genotoxic impurity 2-methyl-6-nitro aniline in telmisartan API. J Appl Pharm Sci 2020;10:92-6

38. Ramachandra B. Development of impurity profiling methods using 
modern analytical techniques. Crit Rev Anal Chem 2017;47:24-36.

39. Baldwin S, Bristow T, Ray A, Rome K, Sanderson N, Sims M, et al. Applicability of GC/quadrupole-orbitrap mass spectrometry in support of pharmaceutical research and development. Rapid Commun Mass Spectrom 2016;30:873-80

40. Sun M, Bai L, Liu DQ. A generic approach for the determination of trace hydrazine in drug substances using in situ derivatizationheadspace GC-MS. J Pharm Biomed Anal 2009;49:529-33.

41. Prasannakumar PB, Balamuralidhara V, Gowrav MP, Venkatesh MP. Nitrosamines in Drug substance and drug product: A regulatory challenge. Int J Res Pharm Sci 2020;11:2123-30.

42. Wollein U, Schramek N. Simultaneous determination of alkyl mesilates and alkyl besilates in finished drug products by direct injection GC-MS. Eur J Pharm Sci 2012;45:201-4.

43. Ho TD, Yehl PM, Chetwyn NP, Wang J, Anderson JL, Zhong Q. Determination of trace level genotoxic impurities in small molecule drug substances using conventional headspace gas chromatography with contemporary ionic liquid diluents and electron capture detection. J Chromatogr A 2014;1361:217-28.

44. Raghavender RS, Hussain RK, Narendra KM, Madhava RP, Venkata RR, Sharma HK. A validated GC-MS method for the determination of genotoxic impurities in divalproex sodium drug substance. J Chromatogr Sci 2019:57:101-7.

45. Chen L, Zhang W, Hu S. Determination of genotoxic epoxide at trace level in drug substance by direct injection GC-MS. J Pharm Biomed Anal 2017;146:103-8.

46. Ahirrao VK, Jadhav RA, Rane VP, Bhamare HR, Yeole RD. Time dependent selected reaction monitoring based GC-MS/MS method for estimation of genotoxic impurities in new antibacterial agent alalevonadifloxacin mesylate. J Anal Sci Technol 2020;11:2-9.

47. Anerao A, Patil B, Pradhan N. Determination of residual dimethyl sulfate in methoxsalen drug substance by pre-column derivatization with static headspace gas chromatography. Int J Pharm Pharm Sci 2018;10:84-9.
48. World Health Organization. Update on Nitrosamine Impurities, EMP/ RHT/Information Note-nitrosamine Impurities. Geneva: World Health Organization; 2019. Available from: https://www.who.int/medicines/ publications/drugalerts/InformationNoteNitrosamine-impurities Nov2019.pdf. [Last accessed on 2020 Jun 18].

49. European Medicines Agency. To Review Ranitidine Medicines Following the Detection of NDMA, EMA/503622/2019. Netherlands: European Medicines Agency; 2019. Available from: https://www. ema.europa.eu/en/documents/press-release/ema-review-ranitidinemedicines-following-detection-ndma_en.pdf. [Last accessed on 2020 Jun 18].

50. European Medicines Agency. Information on Nitrosamines for Marketing Authorization Holders, EMA/189634/2019. European Medicines Agency; 2019. Available from: https://www.ema.europa. $\mathrm{eu} / \mathrm{en} /$ documents/referral/nitrosamines-emea-h-a53-1490-informationnitrosamines-marketing-authorisation-holders_en.pdf. [Last accessed on 2020 Jun 18].

51. Charrois JW, Arend MW, Froese KL, Hrudey SE. Detecting $\mathrm{N}$-nitrosamines in drinking water at nanogram per liter levels using ammonia positive chemical ionization. Environ Sci Technol 2004;38:4835-41.

52. Kodamatani H, Roback SL, Plumlee MH, Ishida KP, Masunaga H, Maruyama $\mathrm{N}$, et al. An inline ion-exchange system in a chemiluminescence-based analyzer for direct analysis of $\mathrm{N}$-nitrosamines in treated wastewater. J Chromatogr A 2018;1553:51-6.

53. Mullett WM, Levsen K, Borlak J, Wu J, Pawliszyn J. Automated in-tube solid-phase microextraction coupled with HPLC for the determination of N-nitrosamines in cell cultures. Anal Chem 2002;74:1695-701.

54. Qian Y, Wu M, Wang W, Chen B, Zheng H, Krasner SW, et al. Determination of 14 nitrosamines at nanogram per liter levels in drinking water. Anal Chem 2015;87:1330-6.

55. Sieira BJ, Carpinteiro I, Rodil R, Quintana JB, Cela R. Determination of N-nitrosamines by gas chromatography coupled to quadrupole-timeof-flight mass spectrometry in water samples. Separations 2020;7:1-12. 